\title{
RELIABLE SIMULATION WITH INPUT UNCERTAINTIES USING AN INTERVAL-BASED APPROACH
}

\author{
Ola Ghazi Batarseh \\ Department of Industrial Engineering \& \\ Management Systems \\ 4000 Central Florida Blvd. \\ Orlando, FL 32816, U.S.A.
}

\author{
Yan Wang \\ Department of Industrial Engineering \& \\ Management Systems \\ 4000 Central Florida Blvd. \\ Orlando, FL 32816, U.S.A.
}

\begin{abstract}
Uncertainty associated with input parameters and models in simulation has gained attentions in recent years. The sources of uncertainties include lack of data and lack of knowledge about physical systems. In this paper, we present a new reliable simulation mechanism to help improve simulation robustness when significant uncertainties exist. The new mechanism incorporates variabilities and uncertainties based on imprecise probabilities, where the statistical distribution parameters in the simulation are intervals instead of precise real numbers. The mechanism generates random interval variates to model the inputs. Interval arithmetic is applied to simulate a set of scenarios simultaneously in each simulation run. To ensure that the interval results bound those from the traditional real-valued simulation, a generic approach is also proposed to specify the number of replications in order to achieve the desired robustness. This new reliable simulation mechanism can be applied to address input uncertainties to support robust decision making.
\end{abstract}

\section{INTRODUCTION}

Modeling and simulation tools are valuable to assess complex systems when analytical approaches are not possible. We are usually interested in studying the behavior of complex systems under total uncertainty. Total uncertainty is composed of two components:

- Variability: it is due to the inherent randomness in the system. In literature, variability is also referred to as stochastic uncertainty, aleatory uncertainty, and irreducible uncertainty. This component is irreducible even by additional measurements. The typical representation of variability is based on probability distributions.

- Uncertainty: it is due to lack of perfect knowledge or enough information about the system. Uncertainty is also known as epistemic uncertainty, reducible uncertainty, and model form uncertainty. Since the uncertainty is caused by the lack of information about the system, it can be reduced by increasing our knowledge to fill the information gap.

In the traditional simulation mechanism, probability distributions with deterministic parameters represent the variability of processes. This assumes that parameters of input distributions are known with certitude. The representation does not capture the total uncertainty because it ignores the uncertainty component in simulation. Uncertainties in simulation have different sources. For instance, the parameters of probability distributions may be uncertain when the sample size of data for input analysis is small or when the measurement errors and the quality associated with the collected data cannot be ignored. When data are not available, experts usually give judgments, which are subjective and can be inconsistent. Other contributors of uncertainties include lack of information about the dependency among factors and variables, as well as unknown time dependency of these factors. Uncertainties may also come from the simulation model itself due to partial knowledge about the physical system.

Some argue that since the data itself can never be quantified with absolute certainty, thus uncertainty is subjective. However, when uncertainty is significant in making decisions, it must be quantified and incorporated into simulations. And it must be interpreted through simulation predictions, regardless the source of it. The two components of the total uncertainty need to be represented explicitly if simulation is used in risk analysis and safety assessment, where decisions are made based on simulation results. Neglecting the uncertainty component may lead to decisions that are not robust.

This research is to develop a reliable simulation mechanism to represent the total uncertainty of systems under study. A simulation mechanism is reliable if its solution is both complete and sound with respect to uncertainties. A complete solution includes all possible occurrences. A sound solution does not include impossible occurrences. We propose a new simulation mechanism based on interval-valued probabilities, which are imprecise probabilities with interval parameters. For ex- 


\section{Batarseh and Wang}

ample, an interval exponential distribution is represented as $\exp ([\underline{\lambda}, \bar{\lambda}])$ where the rate parameter is an interval. The goal of reliable simulation is to incorporate uncertainties and support robust decision makings. In this approach, probabilistic distributions represent variabilities, and intervals capture uncertainties. The degree of uncertainty measures the level of knowledge or information we acquire about the system. It can be considered as a determining factor of the parameters' interval width and is proportionally related.

We use random interval generators to generate random intervals instead of the traditional real-valued random variates. The outputs of simulations are also intervals. Therefore, calculations of performance measures are based on interval arithmetic. Interval statistics methods are used to report the mean and the standard deviation to provide a concise summary of the results. To ensure that the resultant intervals bound all possible real-valued outputs, a generic approach to determine the number of replications is proposed.

The rest of the paper is organized as follows. In Section 2, we review the related work of uncertainty quantification in simulation and introduce imprecise probability. In Section 3, the random interval variants generating method and interval-based simulation are described. Section 4 shows our approach to determine the number of replications for robustness.

\section{BACKGROUND}

In this section, we review the related work of uncertainty quantification in simulation, including the second order Monte Carlo, Bayesian methods, Delta method, and parametric bootstrapping. Some representations of imprecise probabilities are also introduced.

\subsection{Uncertainty Quantification in Simulation}

Uncertainties have been accounted for in simulation with different approaches. The following subsections summarize the different methods that have been developed.

\subsubsection{Second Order Monte Carlo (SOMC) Simulation}

One of the popular simulation techniques that represent the total uncertainty in simulation is the second order Monte Carlo (SOMC) (Burmaster and Wilson 1996). A second-order probabilistic sensitivity analysis is superimposed on the traditional simulation so that uncertainties are quantified by sampling the parameters of the firstorder probabilistic distributions. SOMC contains two simulation loops. The inner loop is called the variability loop that reflects the natural variability. The outer loop represents the uncertainty of the input parameters of the inner loop.

SOMC is easy to implement. However, the doubleloop simulation increases the number of runs. Thus the computational time increases. In each replication of the outer loop, the simulation output captures one of the possible scenarios associated with the uncertain parameters. As the number of replications increases the simulation robustness increases. However, the analyst does not know how many replications to run to achieve the desired robustness representing all possible scenarios. The additional question that has to be asked is whether the analyst has enough information to select the distributions of the input parameters in the outer loop.

Furthermore, the soundness of the response measurement is guaranteed in SOMC given that the distributions of the input parameters are valid. However, the completeness is not verifiable unless the number of replications for the outer loop increases tremendously.

\subsubsection{Bayesian Methods}

Recently the combination of the Bayesian analysis with simulation to capture model and parameter uncertainties has attracted attentions (Chick 2001). Two types of uncertainties, model uncertainty and parameter uncertainty, can be incorporated in the Bayesian methods. The basic idea is to place a prior distribution on each input parameter in simulation to describe its initial uncertainty. The prior distribution is then updated to a posterior distribution based on the observed data associated with each parameter. The posterior distribution has more precise information about the values of the unknown parameters. The two distributions are used to guide the analyst to collect additional data if needed. After a specified number of data points for each parameter are collected, the simulation experiment is updated and run.

Glynn (1986) first proposed a general Bayesian approach to continuously update input distributions with real-world data. Chick (1997) suggested the applications of Bayesian analysis to a broader range of areas such as input uncertainties, rankings, response surface modeling, and experimental design. The input uncertainty issue was further explored by his continuous work and a simulation replication algorithm was developed (Chick 1999, Chick 2000). Andradóttir and Bier (2000) also proposed the Bayesian analysis approach for input uncertainties and model validation. A Bayesian model average (BMA) method was proposed by Chick (2001). BMA is used when multiple candidate distributions are proposed for a single source of randomness ( $\mathrm{Ng}$. and Chick 2006). The idea of BMA was further improved by Zouaoui and Wilson (2001a, 2001b, 2003, 2004), where the analyst has more control on the number of simulation replications. 


\section{Batarseh and Wang}

Bayesian methods quantify the parameter uncertainty in the simulation response. However, the difficulty of computing the posterior distribution hindered the wide spread of its use. The analyst needs more computational procedures such as Markov chain Monte Carlo simulation or importance sampling to implement this method. The non-generality of the Bayesian approach also reduces its use since it needs to be tailored to each application (Henderson 2003).

\subsubsection{Delta Method}

Cheng and Holland (Cheng 1994, Cheng and Holland 1997, Cheng and Holland 1998) developed a delta method for input uncertainties. The framework was also adopted by Zouaoui and Wilson (2001a, 2001b). The method assumes that the model is known while input parameters are uncertain. The true values of the parameters are estimated by the maximum likelihood estimation (MLE), assuming that the parameters follow a normal distribution.

The total simulation output variance is estimated by two terms. The first term is the simulation variance, and the second term is the input parameter variance. The early work of Cheng and Holland (Cheng 1994, Cheng and Holland 1997, Cheng and Holland 1998) did not include the bias in the mean square error (MSE) of the parameters. The failure of including the bias is substantial in the sense that the simulation output confidence intervals are conservative, hence, the variance is overestimated (Henderson 2003).

Later Cheng and Holland (2004) improved on this method by taking into consideration of the bias in the MSE. Nonetheless, its major disadvantage is in the assumption that the model is known with certainty. Furthermore the performance of this method is not yet known compared to Bayesian and Bootstrap methods (Henderson 2003).

\subsubsection{Bootstrap Approach}

Bootstrapping was developed by Efron (1993). There are two formulations of this technique, parametric and nonparametric. Batron and Schruben (2001) proposed three non-parametric resampling methods to incorporate the error due to input distributions. These methods use empirical distribution functions (EDFs) to model the distribution functions of independent input random variables.

For parametric resampling, Cheng and Holland (1997) utilized the bootstrap idea to quantify the effect of input parameter uncertainty for the parametric formulation. Using the available information, the parameters are first estimated by the MLE. The estimates are used to draw a new sample of the observations. This process is repeated $B$ times obtaining $B$ estimates to the input parameters (Cheng and Holland 2004).
The use of percentile confidence interval is recommended in the bootstrapping method in the absence of simulation uncertainty. Its use assumes that the statistics of interest are computed deterministically by the resampling methods. When simulation uncertainty is present, percentile confidence intervals are based on a convolution of the input uncertainty and simulation uncertainty. In the bootstrapping simulation method, it is impossible to separate these forms of uncertainty. Hence, it is still not clear how these intervals behave (Henderson 2003).

\subsection{Imprecise Probability}

In this paper, our proposed reliable simulation mechanism is based on imprecise probability. Instead of a precise value of the probability $P(E)=p$ associated with an event $E$, a pair of lower and upper probabilities $P(E)=[\underline{p}, \bar{p}]$ are used to quantify the uncertainty.

There are many representations of imprecise probabilities. For example, the Dempster-Shafer evidence theory (Dempster 1967, Shafer 1990) characterizes uncertainties as discrete probability masses associated with a power set of values. Belief-Plausibility pairs are used to measure likelihood. The behavioral imprecise probability theory (Walley 1991) models behavioral uncertainties with the lower prevision (supremum acceptable buying price) and the upper prevision (infimum acceptable selling price). A random set (Malchanov 2005) is a multivalued mapping from the probability space to the value space. The possibility theory (Dubois and Prade 1988) provides an alternative to represent uncertainties with Necessity-Possibility pairs. Probability bound analysis (Ferson et al. 2003) captures uncertain information with pboxes which are pairs of lower and upper probability distributions. F-probability (Weichselberger 2000) incorporates intervals into probability values which maintains the Kolmogorov properties. Fuzzy probability (Möller and Beer 2004) considers probability distributions with fuzzy parameters. A cloud (Neumaier 2004) is a fuzzy interval with an interval-valued membership, which is a combination of fuzzy sets, intervals, and probability distributions. Recently, an imprecise probability with generalized interval form (Wang 2008) was also proposed, where the probabilistic calculus structure is simplified based on the algebraic closure properties of the Kaucher arithmetic (Kaucher 1980) for generalized intervals.

Imprecise probability captures the total uncertainty and represents its two components quantitatively. It provides a concise form to improve the robustness of simulation without the traditional sensitivity analysis alike operations. 


\section{RELIABLE SIMULATION MECHANISM}

The idea of the proposed new simulation mechanism is to compute and simulate based on intervals instead of the traditional floating-point numbers. In the traditional simulation, if it is possible to collect data for an input random variable of interest, these data are used in fitting a theoretical distribution with certitude. However, when the limitations mentioned in Section 1 are encountered and input uncertainties cannot be ignored, one needs to find new approaches to extend the current fitting method based on precise parameters.

In the proposed new simulation mechanism, we incorporate input uncertainty factors by using intervals as input parameters. Based on imprecise probability, this mechanism allows us to simulate from a set of probabilities at the same time and simultaneously consider a range of scenarios. Three major components of the new simulation mechanism, random interval variate generation, interval-based uncertainty propagation, and interval statistics, are described in Sections 3.1, 3.2, and 3.3 respectively.

\subsection{Random Interval Variate Generation}

The parameters of statistical distributions in reliable simulation are intervals instead of real numbers. For example, an interval exponential distribution expo ([2,4]) is used instead of expo (3). With this interval representation of parameters, the degree of uncertainties is captured by intervals' widths. The larger the parameter interval width is, the less knowledge we have about this parameter. As a result, the cumulative distribution function $(c d f)$ associated with an input is no longer one crispy curve. Instead, we have a pair of $c d f$ 's corresponding to the lower and upper bounds.

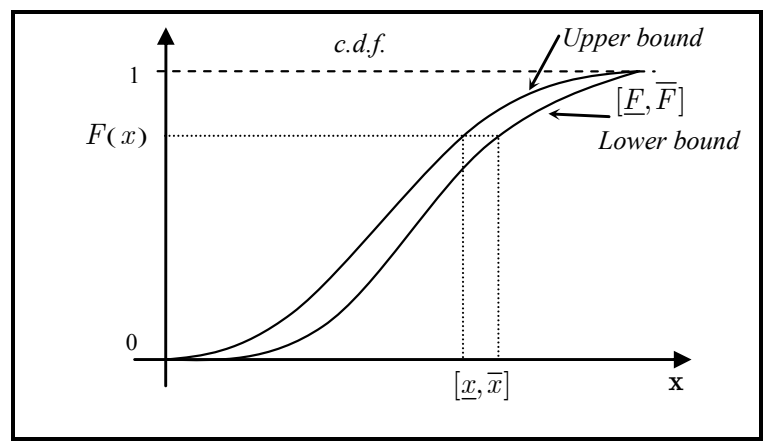

Figure 1: Upper and lower cumulative distribution functions are used in the inverse transform to generate random intervals

Figure 1 illustrates the random interval variate generating method with the inverse transform. At a certain value of cumulative probability $F(x)$, the generated random variate is $[\underline{x}, \bar{x}]$ where $\underline{x}$ and $\bar{x}$ are the lower and upper bounds of the random interval variate respectively. The upper and lower bounds of $c d f$ in Figure 1 can also be read in a second way. For a value of a random variable $x$, the cumulative probability is represented by an interval probability $[\underline{F}(x), \bar{F}(x)]$. These two representations of uncertainties are equivalent. With this approach, random intervals capture the uncertainty while the variability is represented by the probabilistic properties of the random interval population.

\subsection{Uncertainty Propagation}

The propagation of uncertainties during simulation is based on interval arithmetic. Specifically, the Kaucher interval arithmetic operations (Kaucher 1980) are used in calculation. We implement the random interval variate generation as well as the Kaucher interval arithmetic operations in a Java-based object-oriented simulation package, JSim.

An example of a simple linear system shown in Figure 2 is used to illustrate the simulation process. The random numbers used in simulation are intervals, which are generated according to the corresponding interval probabilistic distributions. The initial state of an entity is determined by a Source, which creates entities based on statistical distributions of inter-arrival times. The entity starts its life cycle in the system with the arrival time represented by an interval $[\underline{a}, \bar{a}]$. Then each entity passes through the required Stations in its life cycle and stays for a time period of $\left[s_{i}, \overline{s_{i}}\right]$ for the $i$-th station. After an entity's life cycle ends, it is routed to a Sink.

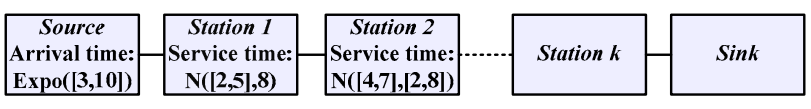

Figure 2: Simple Linear System Based on Intervals

In this interval-based simulation, the calculations of the performance measures are based on interval arithmetic. As a simple example to illustrate, the total time of an entity in the system is the total time spent from Station 1 to Station $k$ and is computed as $\left[\underline{s}_{1}+\ldots+\underline{s}_{k}, \bar{s}_{1}+\ldots+\bar{s}_{k}\right]$.

One important part of the interval-based simulation mechanism is the simulation clock. The partial order between two intervals is more complex than real numbers. In other words, the less than or equal to relationship between $[\underline{a}, \bar{a}]$ and $[\underline{b}, \bar{b}]$ can be defined in several ways. To simplify the implementation, we still use the real-valued simulation time to keep track of events. If the time of the next event is a random interval $[\underline{t}, \bar{t}]$, the simulation clock 


\section{Batarseh and Wang}

is advanced based on a random variable $t$ that is uniformly distributed within $[\underline{t}, \bar{t}]$, i.e. $t \sim U(t, \bar{t})$. For instance, if the current simulation time is $t_{0}$ and the interarrival time of entities are randomly generated as intervals $\left[\underline{a}_{i}, \bar{a}_{i}\right]$ 's, these arrival events are assumed to be uniformly distributed within $\left[\underline{a}_{i}, \bar{a}_{i}\right]$ 's and random numbers are sampled from $\left[\underline{a}_{i}, \bar{a}_{i}\right]$ 's to specify their arrival times. The clock advances based on this real-valued time $a_{i} \sim U\left(\left[\underline{a}_{i}, \bar{a}_{i}\right]\right)$. All other events that trigger the clock to advance are modeled in a similar vein.

\subsection{Interval Statistics}

The simulation outputs for performance measures are intervals. Therefore statistics based on intervals should be used to draw conclusions from the sample data. The most frequently used statistical summaries are the mean (average) and the variance (standard deviation). Section 3.3.1 shows how to compute the arithmetic mean of interval data and Section 3.3.2 discusses algorithms used to compute bounds on the variance for an interval data set.

\subsubsection{Mean}

The mean of a set of intervals $\left\{\left[\underline{x}_{i}, \bar{x}_{i}\right] \in \mathrm{IR} \mid \underline{x}_{i} \leq \bar{x}_{i}, \underline{x}_{i} \in \mathrm{R}, \bar{x}_{i} \in \mathrm{R}\right\}$ is also an interval. It should include the smallest possible and the largest possible means. which can be calculated from any possible real number $x_{i} \in\left[\underline{x}_{i}, \bar{x}_{i}\right]$. Because the formula to calculate mean is a monotone function, the lower bound of the interval mean is just the average of the left endpoints $\underline{x}_{i}$ 's, and the upper bound is the average of the right endpoints $\bar{x}_{i}$ 's (Granvilliers et al. 2003). Therefore the arithmetic mean of random intervals is given by

$$
[\underline{\mu}, \bar{\mu}]=\left[\frac{1}{N} \sum_{i=1}^{N} \underline{x_{i}}, \frac{1}{N} \sum_{i=1}^{N} \overline{x_{i}}\right]
$$

where $N$ is the sample size of random intervals.

\subsubsection{Variance}

One of the important measures of data dispersion is variance. Computing the range for the variance $[\underline{V}, \bar{V}]$ for a set of intervals is an NP-hard problem (Granvilliers et al. 2003). Kreinovich et al. proposed algorithms to obtain the bounds on the variance (Ferson et al. 2007, Granvilliers et al. 2003, Xiang et al. 2007). For computing the lower bound of the variance $V$, there exists computationally feasible algorithms. It was found that $\underline{V}$ can be computed in $O(n \log (n))$ computational steps for $n$ interval data points $\left[\underline{x}_{i}, \bar{x}_{i}\right]$. On the other hand, computing the upper bound of the variance $\bar{V}$ requires computational effort that grows exponentially with the number of intervals in the data set (Granvilliers et al. 2003). However, for several special cases such as when intervals are not overlapped and there is no interval completely nested in another one, $O(n \log (n))$ and linear time algorithms are available to compute $\bar{V}$ (Ferson et al. 2007, Xiang et al. 2007).

\section{SIMULATION ROBUSTNESS MEASURE}

In order to have reliable and robust simulations, interval outputs produced by the interval input parameters should include those possible scenarios. That is, we need to have a certain level of confidence that the lower and upper $c d f$ 's of interval outputs bound the output $c d f$ obtained from any bounded real-valued input parameters.

Three factors play the roles to achieve the above mentioned enclosure effect. The first is the interval width of interval input parameters. As the width of the interval parameters increases, the probability that the real-valued $c d f$ is enclosed by the lower and upper $c d f$ 's increases. However, if the interval width of inputs increases more than needed, the widths of the simulation output intervals will be over-estimated. The second factor is the value of the input real-valued parameters with respect to the bounds of the interval parameters. As the value of the real-valued parameter gets closer to either bound of the interval parameter, the probability of including the realvalued $c d f$ between the lower and upper $c d f$ 's decreases. The third factor is the number of replications used in simulation. As the number of replications increases, the probability of enclosure will increase. The most reliable output is achieved by having an infinite number of replications. But obviously, this is impractical.

In this section, we derive an algebraic relationship between the number of replications and the accuracy of the estimate measured by the probability of enclosure. The relationship is used to determine the minimum number of replications necessary for a prefixed value of accuracy. The relationship can be also used inversely. If the analyst can afford a certain number of replications, this relationship provides the width of the interval parameter to be used to achieve a certain level of accuracy.

The approach used to find the number of replications $n$ is based on the probability of having an assumed realvalued random variable $x$ bounded by the corresponding random interval $[\underline{x}, \bar{x}]$ at any cumulative probability $p$ in $c d f$. The goal is to achieve the probability of enclosure

$$
P(x \in[\underline{x}, \bar{x}]) \geq 1-\alpha
$$




\section{Batarseh and Wang}

where $x$ is the random variable if the simulation is run from any real-valued parameter bounded by the interval parameter.

There are three possible locations of $x$ with respect to $[\underline{x}, \bar{x}]: x<\underline{x}, x>\bar{x}$, and $\bar{x} \leq x \leq \bar{x}$. The last location is the desirable one. If the independence of the lower and upper bounds is assumed, the probability in (2) can be rewritten as

$$
P(\underline{x} \leq x \leq \bar{x})=P(\underline{x} \leq x) \times(1-P(x \leq \bar{x}))
$$

Order statistics is used to ensure that the probability in (3) at any cumulative probability $p$ is at least $1-\alpha$. If the real-valued variables are ordered as $x_{(1)}, x_{(2)}, \ldots ., x_{(n)}$, the corresponding value of $p$ associated with the $r^{\text {th }}$ ordered observation is given by $(r-0.5) / n$. The sampling distribution of the transformed order statistics $c d f$ is given by $G_{r}(x) . G_{r}(x)$ is interpreted as the probability that at least $r$ observations in the sample do not exceed $x$ and can be calculated as (Stuart 1987)

$$
G_{r}(x)=\sum_{j=r}^{n}\left[\left(\begin{array}{l}
n \\
j
\end{array}\right)(F(x))^{j}(1-F(x))^{n-j}\right]
$$

where $F(x)$ is the $c d f$ of the random variable $x$.

Based on the ordered statistics sampling distribution, the probability of having the $r^{\text {th }}$ random variable $x_{r}$ between the $r^{\text {th }}$ bounds of the interval random variable $\left[\underline{x}_{r}, \bar{x}_{r}\right]$ is given by

$$
P\left(\underline{x}_{r} \leq x_{r} \leq \bar{x}_{r}\right)=\underline{G}\left(x_{r}\right) \times\left(1-\bar{G}\left(x_{r}\right)\right)
$$

where $\underline{G}\left(x_{r}\right)$ and $\bar{G}\left(x_{r}\right)$ are the lower and the upper sampling distribution. Substituting (4) in (5) we receive

$$
\begin{aligned}
& P\left(\underline{x}_{r} \leq x_{r} \leq \bar{x}_{r}\right)=\left(\sum_{j=r}^{n}\left[\left(\begin{array}{l}
n \\
j
\end{array}\right)\left(\bar{F}\left(x_{r}\right)\right)^{j}\left(1-\bar{F}\left(x_{r}\right)\right)^{n-j}\right]\right) \times \\
& \left.\left(1-\sum_{j=r}^{n}\left[\left(\begin{array}{l}
n \\
j
\end{array}\right)\left(\underline{F\left(x_{r}\right)}\right)\right)^{j}\left(1-\underline{F}\left(x_{r}\right)\right)^{n-j}\right]\right)
\end{aligned}
$$

The probability in (6) can be used for any probabilistic distribution function by replacing the lower and the upper cumulative distribution functions $\underline{F}(x)$ and $\bar{F}(x)$ respectively with the corresponding distribution form. This relationship is used to determine the number of replications needed to ensure that the probability in (2) is achieved.

Here we derive the specific form of (6) for the exponential distribution to demonstrate the use of the derived relationship. Assume a stochastic process follows an exponential distribution with an estimated real-valued mean of $\beta$. An interval exponential distribution with the mean of $[\underline{\beta}, \bar{\beta}]$ is used to enclose the real-valued $c d f$, where $\beta \in[\beta, \bar{\beta}]$. The upper bound $c d f$ is associated with $\underline{\beta}$ and the lower bound $c d f$ is with $\bar{\beta}$. Substituting the exponential cumulative distribution functions

$$
\begin{aligned}
& \underline{F}(x)=1-e^{-x / \bar{\beta}} \\
& \bar{F}(x)=1-e^{-x /} \underline{\beta}
\end{aligned}
$$

into (6) we receive

$$
\begin{aligned}
& P\left(\underline{x}_{r} \leq x_{r} \leq \bar{x}_{r}\right)=\left(\sum_{j=r}^{n}\left[\left(\begin{array}{l}
n \\
j
\end{array}\right)\left(1-e^{-x_{r} / \beta}\right)^{j}\left(e^{-x_{r} / \underline{\beta}}\right)^{n-j}\right]\right) \times \\
& \left(1-\sum_{j=r}^{n}\left[\left(\begin{array}{l}
n \\
j
\end{array}\right)\left(1-e^{-x_{r} / \bar{\beta}}\right)^{j}\left(e^{-x_{r} / \bar{\beta}}\right)^{n-j}\right]\right)
\end{aligned}
$$

where $r$ is the order of the observation associated with the percentile $p$. With the inverse transform of exponential distribution

$$
x_{r}=-\beta \ln (1-p)
$$

$x_{r}$ is calculated at the cumulative probability $p$. (7) now can be approximated as

$$
\begin{aligned}
& P\left(x_{r} \leq x_{r} \leq x_{r}\right)=\left(\sum_{\sum_{j=r}^{n}}\left[\begin{array}{l}
\left(\begin{array}{l}
n \\
j
\end{array}\right)\left(1-\left(1-\frac{r-0.5}{n}\right)^{\beta /} \underline{\beta}^{j}\right. \\
\left.\times\left(1-\frac{r-0.5}{n}\right)^{\beta /}\right)^{n-j}
\end{array}\right]\right) \\
& \times 1-\left(\sum_{j=r}^{n}\left[\begin{array}{l}
\left(\begin{array}{l}
n \\
j
\end{array}\right)\left(1-\left(1-\frac{r-0.5}{n}\right)^{\beta / \beta}\right)^{j} \\
\left.\left.\times\left(\left(1-\frac{r-0.5}{n}\right)^{\beta / \beta}\right)^{n-j}\right]\right)
\end{array}\right)\right)
\end{aligned}
$$

(8) gives the probability that the random real variable $x$ of order $r$ is bounded between the random interval variable $[\underline{x}, \bar{x}]$ when both are exponentially distributed. Setting the probability to a desired value, we can estimate the number of replications $n$ at each percentile.

We solve the equation in (8) numerically for three ratios of $[\beta / \beta, \bar{\beta} / \beta]$ as $[0.9,1.1],[0.8,1.2]$, and $[0.6,1.4]$ and construct Tables 1, 2 and 3 respectively. The tables can be used based on the ratios between the bounds of the interval mean and the real-valued mean regardless the absolute values of the means. The numbers of replications were calculated to achieve the confidence levels (CLs) of $90 \%$ and $95 \%$. For Table 1 the number of replications was calculated only at $90 \% \mathrm{CL}$. The number of replications needed at $90 \% \mathrm{CL}$ is very large in general due to the narrow interval of $[0.9,1.1]$. The numbers of replications for 


\section{Batarseh and Wang}

95\% CL are even greater than the corresponding ones for $90 \% \mathrm{CL}$. It is noticed that as the interval width increases the number of replications for simulation decreases at the same percentile yielding the same probability of enclosure.

Table 1: Calculation of the numbers of replications needed for the interval ratio $[\underline{\beta} / \beta, \bar{\beta} / \beta]=[0.9,1.1]$

\begin{tabular}{c|c}
\hline$r$ & $\alpha=0.1$ \\
\hline 1 & $>1030 \max (p)=0.271$ \\
\hline 2 & $>1030 \max (p)=0.300$ \\
\hline 3 & $>1030 \max (p)=0.317$ \\
\hline 4 & $>1030 \max (p)=0.330$ \\
\hline 5 & $>1030 \max (p)=0.342$ \\
\hline 6 & $>1030 \max (p)=0.352$ \\
\hline 7 & $>1030 \max (p)=0.362$ \\
\hline 8 & $>1030 \max (p)=0.371$ \\
\hline 9 & $>1030 \max (p)=0.379$ \\
\hline 10 & $>1030 \max (p)=0.387$ \\
\hline 20 & $>1030 \max (p)=0.452$ \\
\hline 30 & $>1030 \max (p)=0.501$ \\
\hline 40 & $>1030 \max (p)=0.542$ \\
\hline 50 & $>1030 \max (p)=0.578$ \\
\hline 60 & $>1030 \max (p)=0.609$ \\
\hline 70 & $>1030 \max (p)=0.638$ \\
\hline 80 & $>1030 \max (p)=0.664$ \\
\hline 90 & $>1030 \max (p)=0.687$ \\
\hline 100 & $>1030 \max (p)=0.708$ \\
\hline 110 & $>1030 \max (p)=0.728$ \\
\hline 120 & $>1030 \max (p)=0.746$ \\
\hline 130 & $>1030 \max (p)=0.762$ \\
\hline 140 & $>1030 \max (p)=0.777$ \\
\hline 150 & $>1030 \max (p)=0.792$ \\
\hline 160 & $>1030 \max (p)=0.804$ \\
\hline 170 & $>1030 \max (p)=0.817$ \\
\hline 180 & $>1030 \max (p)=0.828$ \\
\hline 190 & $>1030 \max (p)=0.838$ \\
\hline 200 & $>1030 \max (p)=0.848$ \\
\hline 250 & $>1030 \max (p)=0.888$ \\
\hline 300 & 433 \\
\hline 350 & 418 \\
\hline 400 & 488 \\
\hline 450 & \\
\hline & \\
\hline 150
\end{tabular}

In the calculation, we stopped when $n$ is greater than 1030 because the program reaches its computational limit of calculating the large $n$ due to the factorial. In the tables, ( $>1030)$ indicates the limit is reached. The maximum bounding probability when $n=1030$ is also given in the tables. The transition from $>1030$ to three or two decimals of replication numbers shows how affordable it is to reach the completeness of the solution in these orders of $r$. For the small orders of observations, the number of replications required for a specified CL is very large. It shows the difficulty of enclosing the real-valued $c d f$ at small orders of $r$ for small interval widths compared to large interval widths. For the very large orders of observations, the number of replications also starts to increase.
This is due to the narrow width of the $c d f$ bounds at the high cumulative probability as the $c d f$ curves become flatter.

Table 2: Calculation of the numbers of replications needed for the interval ratio $[\beta / \beta, \bar{\beta} / \beta]=[0.8,1.2]$

\begin{tabular}{|c|c|c|}
\hline$r r$ & $\alpha=0.1$ & $\alpha=0.05$ \\
\hline 1 & $>1030 \max (p)=0.306$ & $>1030 \max (p)=0.306$ \\
\hline 2 & $>1030 \max (p)=0.360$ & $>1030 \max (\mathrm{P})=0.360$ \\
\hline 3 & $>1030 \max (p)=0.395$ & $>1030 \max (p)=0.395$ \\
\hline 4 & $>1030 \max (p)=0.424$ & $>1030 \quad \max (p)=0.424$ \\
\hline 5 & $>1030 \max (p)=0.448$ & $>1030 \max (p)=0.448$ \\
\hline 6 & $>1030 \max (p)=0.470$ & $>1030 \max (p)=0.470$ \\
\hline 7 & $>1030 \max (p)=0.491$ & $>1030 \max (p)=0.491$ \\
\hline 8 & $>1030 \max (p)=0.509$ & $>1030 \max (p)=0.509$ \\
\hline 9 & $>1030 \max (p)=0.526$ & $>1030 \max (p)=0.526$ \\
\hline 10 & $>1030 \max (p)=0.542$ & $>1030 \max (p)=0.542$ \\
\hline 20 & $>1030 \max (p)=0.666$ & $>1030 \max (p)=0.666$ \\
\hline 30 & $>1030 \max (p)=0.748$ & $>1030 \max (p)=0.748$ \\
\hline 40 & $>1030 \max (p)=0.806$ & $>1030 \max (p)=0.806$ \\
\hline 50 & $>1030 \max (p)=0.849$ & $>1030 \max (p)=0.849$ \\
\hline 60 & $>1030 \max (p)=0.882$ & $>1030 \max (p)=0.882$ \\
\hline 70 & 143 & $>1030 \max (p)=0.906$ \\
\hline 80 & 105 & $>1030 \max (p)=0.926$ \\
\hline 90 & 106 & $>1030 \max (p)=0.940$ \\
\hline 100 & 112 & 273 \\
\hline 110 & 120 & 161 \\
\hline 120 & 129 & 152 \\
\hline 130 & 138 & 154 \\
\hline 140 & 147 & 160 \\
\hline 150 & 156 & 167 \\
\hline
\end{tabular}

Table 3: Calculation of the numbers of replications needed for the interval ratio $[\underline{\beta} / \beta, \bar{\beta} / \beta]=[0.6,1.4]$

\begin{tabular}{c|c|c}
\hline$r$ & $\alpha=0.1$ & $\alpha=0.05$ \\
\hline 1 & $>1030 \max (p)=0.396$ & $>1030 \max (p)=0.396$ \\
\hline 2 & $>1030 \max (p)=0.506$ & $>1030 \max (p)=0.506$ \\
\hline 3 & $>1030 \max (p)=0.577$ & $>1030 \max (p)=0.577$ \\
\hline 4 & $>1030 \max (p)=0.631$ & $>1030 \max (p)=0.631$ \\
\hline 5 & $>1030 \max (p)=0.675$ & $>1030 \max (p)=0.675$ \\
\hline 6 & $>1030 \max (p)=0.712$ & $>1030 \max (p)=0.712$ \\
\hline 7 & $>1030 \max (p)=0.743$ & $>1030 \max (p)=0.743$ \\
\hline 8 & $>1030 \max (p)=0.769$ & $>1030 \max (p)=0.769$ \\
\hline 9 & $>1030 \max (p)=0.792$ & $>1030 \max (p)=0.792$ \\
\hline 10 & $>1030 \max (p)=0.811$ & $>1030 \max (p)=0.811$ \\
\hline 20 & 29 & $>1030 \max (p)=0.920$ \\
\hline 30 & 32 & 46 \\
\hline 40 & 42 & 53 \\
\hline 50 & 51 & 62 \\
\hline 60 & 61 & 91 \\
\hline 70 & 71 & 101 \\
\hline 80 & 81 &
\end{tabular}

The calculations in Tables 1, 2 and 3 are based on the exponential distributions, which can be extended to other distributions by substituting the appropriate $c d f$ 's in (6) for interval distributions and the inverse function of $c d f$ 


\section{Batarseh and Wang}

for the real-valued distribution. For instance, when the normal distributions are assumed for the real-valued variables $x \sim N\left(\mu, \sigma_{1}\right)$ and the interval random variables $[\underline{x}, \bar{x}] \sim N\left([\underline{\mu}, \bar{\mu}], \sigma_{2}\right)$, the normal $c d f$ used for the upper and the lower bounds is given by

$$
\begin{aligned}
& \bar{F}(x)=\frac{1}{2}\left[1+\operatorname{erf}\left(\frac{x-\underline{\mu}}{\sigma_{2} / \sqrt{2}}\right)\right] \\
& \underline{F}(x)=\frac{1}{2}\left[1+\operatorname{erf}\left(\frac{x-\underline{\mu}}{\sigma_{2} / \sqrt{2}}\right)\right]
\end{aligned}
$$

where the error function erf is given by

$$
\operatorname{erf}(x)=\frac{2}{\sqrt{\pi}} \int_{0}^{x} e^{-t^{2}} d t
$$

Substituting the $c d f$ of normal distributions in (6) we have

$$
\begin{aligned}
& \left.P\left(\underline{x}_{r} \leq x_{r} \leq \bar{x}_{r}\right)=\left(\begin{array}{c}
\sum_{j=r}^{n}\left[\begin{array}{l}
n \\
j
\end{array}\right)\left(\frac{1}{2}+\frac{1}{2} \operatorname{erf}\left(\frac{x_{r}-\underline{\mu}}{\sigma_{2} / \sqrt{2}}\right)\right)^{j} \\
\times\left(\frac{1}{2}-\frac{1}{2} \operatorname{erf}\left(\frac{x_{r}-\underline{\mu}}{\sigma_{2} / \sqrt{2}}\right)\right)^{n-j}
\end{array}\right]\right) \\
& \times\left(1-\sum_{j=r}^{n}\left[\begin{array}{l}
\left(\begin{array}{c}
n \\
j
\end{array}\right)\left(\frac{1}{2}+\frac{1}{2} \operatorname{erf}\left(\frac{x_{r}-\bar{\mu}}{\sigma_{2} / \sqrt{2}}\right)\right)^{j} \\
\left.\left(\frac{1}{2}-\frac{1}{2} \operatorname{erf}\left(\frac{x_{r}-\bar{\mu}}{\sigma_{2} / \sqrt{2}}\right)\right)^{n-j}\right]
\end{array}\right)\right.
\end{aligned}
$$

$x_{r}$ is calculated from the inverse transform of normal distribution as

$$
x_{r}=\mu+\sqrt{2} \sigma_{1} \operatorname{erf}^{-1}\left(\frac{2 r-1}{n}-1\right)
$$

where $\operatorname{erf}^{-1}$ is the inverse of the error function. The normal distribution $c d f$ and its inverse are not available in closed form. Their computation requires the use of numerical procedures, which are widely available in software for statistical modeling.

\section{CONCLUDING REMARKS}

In this paper we proposed an interval based reliable simulation mechanism, which simulates based on intervals instead of floating-point numbers. A random interval variate generating method was used to generate random intervals from a pair of $c d f$ 's corresponding to the lower and upper bounds of the input parameter. We also proposed the method to determine the number of replications for simulation robustness and output reliability. This me- chanism differentiates input uncertainty from variability. It is implemented in a Java-based object-oriented simulation package.

The future work includes the systematic study of the real-valued time in simulation clock. We need to investigate how this affects the simulation results when the sequence of events will be different among replications. We also need to study the fitting of other distribution functions with interval parameters, e.g. Gamma and Lognormal functions. How to interpret the meanings of the collected interval statistics requires further investigations.

\section{REFERENCES}

Andradóttir, S. and Bier, V.M. 2000. Applying Bayesian ideas in simulation. Simulation Practice and Theory, 8 (3-4), 253-280.

Barton, R. and Schruben, L. 2001. Resampling methods for input modeling. In Proceedings of the 2001 Winter Simulation Conference, ed. A.B. Peters, J.S. Smith, D. J. Medeiros, and M. W. Rohrer.

Burmaster, D. and Wilson, A. 1996. An Introduction to Second-Order Random Variables in Human Health Risk Assessments. Human and Ecological Risk Assessment Vol. 2.

Cheng, R. C. H. 1994. Selecting input models. In Proceedings of the 1994 Winter Simulation Conference, ed. J. D. Tew, S. Manivannan, D. A. Sadowski, and A. F. Seila, Institute of Electrical and Electronics Engineers, Piscataway, NJ, 184-191.

Cheng, R.C.H. and Holland, W. 1997. Sensitivity of computer simulation experiments to errors in input data. Statistical Computation and Simulation, 57, 219-241.

Cheng, R.C.H. and Holland, W. 1998. Two-point methods for assessing variability in simulation output. Statistical Computation and Simulation, 60, 183-205.

Cheng, R.C.H. and Holland, W. 2004. Calculation of Confidence Intervals for Simulation Output. $A C M$ Transactions on Modeling and Computer Simulation. Vol. 14, No. 4, 344-362.

Chick, S. E. 1997. Bayesian Analysis for Simulation Input and Output. In Proceedings of the 1997 Winter Simulation Conference, S. Andradóttir, K. J. Healy, D. H. Withers and B. L. Nelson (eds.), Institute of Electrical and Electronics Engineers, Piscataway, New Jersey, 253-260.

Chick, S.E. 1999. Steps to implement Bayesian input distribution selection, In Proceedings of the 1999 Winter Simulation Conference, Farrington, P.A., Nembhard, H.B., Sturrock, D.T. and Evans, G.W. (eds.), Institute of Electrical and Electronics Engineers, Piscataway, NJ, 317-324.

Chick, S. E. 2000. Bayesian methods for simulation. In Proceedings of the 2000 Winter Simulation Conference, ed. J. A. loines, R. R. Barton, K. Kang, and P. 


\section{Batarseh and Wang}

A. Fishwick, Institute of Electrical and Electronics Engineers, Piscataway, NJ, 109-118.

Chick, S. E. 2001. Input distribution selection for simulation experiments: accounting for input uncertainty. Operations Research, 49, 744-758.

Chick, S.E., 2006, Bayesian Ideas for Discrete Event Simulation: Why, What and How, In R.G. Ingalls, M.D. Rossetti, J.S. Smith, B.A. Peters, eds., Proc. Winter Simulation Conference, 96-106

Dempster, A. 1967. Upper and lower probabilities induced by a multi-valued mapping. Annals of Mathematical Statistics, 38(2):325-339.

Dubois, D. and H. Prade. 1988 Possibility Theory: An Approach to Computerized. Processing of Uncertainty, Plenum, New York.

Efron, B. and Tibshirani, R. J. 1982. An Introduction to the bootstrap. Chapman and Hall/CRC.

Ferson, S., Kreinovich, V. Ginzburg, L. Myers D.S., and Sentz, K. 2003. Constructing probability boxes and Dempster-shafer structures. Sandia National Laboratories Technical report SAND2002-4015, Albuquerque, NM.

Ferson, S., Kreinovich, V., Hajagos, J., Oberkampf, W., and Ginzburg, L. 2007. Experimental Uncertainty Estimation and Statistics for Data Having Interval Uncertainty. Sandia National Laboratories Technical report SAND2007-0939, Setauket, New York.

Glynn, P. 1986. Problems in Bayesian analysis of stochastic simulation. In Proceedings of the 1986 Winter Simulation Conference, ed. J. R. Wilson, J. O. Henriksen, and S. D. Roberts, 376-383.

Granvilliers, L. Kreinovich, V. and MÄuller, N. 2003. Novel approaches to numerical software with result verification, In: R. Alt, A. Frommer, R. B. Kearfott, and W. Luther (eds.), Numerical Software with Result Verification, (International Dagstuhl Seminar, Dagstuhl Castle, Germany, Springer Lectures Notes in Computer Science, 2004, Vol. 2991, 274-305.

Henderson, S. 2003 Input Model Uncertainty: Why do We Care and What Should We Do About It? In Proceedings of the 2003 Winter Simulation Conference., ed. S. Chick, P.J. Sanchez, D. Ferrin and D. J. Morrice

Kaucher, E. 1980, Interval analysis in the extended interval space IR. Computing Supplementa, Vol.2, 33-49

Molchanov, I. 2005. Theory of Random Sets. Springer, London.

Möller, B. and Beer M. 2004 Fuzzy Randomness: Uncertainty in Civil Engineering and Computational Mechanics. Springer, Berlin.

Neumaier, A. 2004 Clouds, fuzzy sets, and probability intervals. Reliable Computing, 10(4):249-272.

Ng, S.H., Chick, S.E., 2006, Reducing Parameter Uncertainty for Stochastic Systems, ACM Transactions on Modeling and Computer Simulation, 16(1): 26-51
Shafer, G. A. 1990. Mathematical Theory of Evidence, Princeton University Press, Princeton, NJ.

Stuart, A. and Ord, J. K. 1987. Kendall's Advanced Theory Of Statistics. $5^{\text {th }}$ ed. Vol. 1. New York: Oxford University Press.

Walley, P. 1991. Statistical Reasoning with Imprecise Probabilities, Chapman \& Hall, London.

Wang, Y. 2008. Imprecise probabilities with a generalized interval form. In R.L. Muhanna and R.L. Mullen, eds., Proc. 3rd Int. Workshop on Reliability Engineering Computing (REC'08), Savannah, Georgia, 45-59.

Weichselberger, K. 2000 The theory of intervalprobability as a unifying concept for uncertainty. International Journal of Approximate Reasoning, 24(23), 149-170.

Xiang, G,. Starks S.A, Kreinovich, V. and Longpre, L. 2007. Computing population variance and entropy under interval uncertainty: linear-time algorithms

Zouaoui, F., and J. R. Wilson. 2001a. Accounting for input model and parameter uncertainty in simulation. In Proceedings of the 2001 Winter Simulation Conference, ed. B. A. Peters, J. S. Smith, D. J. Medeiros, and M. W. Rohrer, 29S299. Piscataway, NJ: IEEE.

Zouaoui, F., and J. R. Wilson. 2001b. Accounting for parameter uncertainty in simulation input modeling. In Proceedings of the 2001 Winter Simulation Conference, ed. B. A. Peters, J. S. Smith, D. J. Medeiros, and M. W. ohrer, Institute of Electrical and Electronics Engineers, Piscataway, NJ, 354-363.

Zouaoui, F. and J. R. Wilson. 2003. Accounting for parameter uncertainty in simulation input modeling. IIE Transactions 35, 781-792.

Zouaoui, F. and J. R. Wilson. 2004. Accounting for inputmodel and input-parameter uncertainties in simulation. IIE Transactions 36 (11), 1135-1151

\section{AUTHOR BIOGRAPHIES}

OLA BATARSEH is a Ph.D. student at the Department of Industrial Engineering and Management Systems, University of Central Florida. She received her B.S. from the University of Jordan. Her research interests include robust modeling and simulation.

YAN WANG is an Assistant Professor at the Department of Industrial Engineering and Management Systems, University of Central Florida. He received his B.S. from Tsinghua University, M.S. from Chinese Academy of Sciences, and Ph.D. from the University of Pittsburgh. His research interests include engineering design, modeling, simulation and visualization. 